\title{
Can Adaptive Interfaces Improve the Usability of Mobile Applications?
}

\author{
Janet L. Wesson, Akash Singh, and Bradley van Tonder \\ Department of Computing Sciences, Nelson Mandela Metropolitan University, \\ PO Box 77000+2741 5042323 \\ \{Janet.Wesson, Akash.Singh, Bradley.vanTonder\} @nmmu .ac.za
}

\begin{abstract}
Mobile applications are becoming increasingly widespread and complex. Many of these applications suffer from usability issues, including information overload, screen clutter, lack of task support and limited interaction mechanisms. Adaptive user interfaces (AUIs) have been proposed to address some of these usability issues. The aim of this paper is to investigate how AUIs can improve the usability of mobile applications. This paper discusses several simple types of adaptation that have been shown to yield significant usability benefits for mobile applications. Two case studies are presented to illustrate how an AUI can be incorporated into different types of mobile applications. This paper also discusses the lessons learned from these case studies and presents some implications for designing adaptive systems in the future.
\end{abstract}

Keywords: Adaptive user interfaces, adaptive systems, mobile applications, usability evaluation.

\section{Introduction}

Research has shown that modern information systems suffer from several usability problems (issues). These issues are mostly attributed to the complexity and lack of flexibility of the user interface (UI). Information systems developed for mobile phones also suffer from similar usability issues. The physical constraints of these devices have an impact on screen size and available interaction mechanisms. These constraints contribute to the existence of several usability issues for mobile applications.

Adaptive User Interfaces (AUIs) can provide potential benefits for addressing these usability issues [1]. Adaptation of the UI has been identified as an important aspect to be considered in the design of modern information systems [2]. Adaptation techniques include adapting what information to present (information adaptation), how to present this information (presentation adaptation) and how to interact with this information (interface adaptation).

The aim of this paper is to investigate how AUIs can improve the usability of mobile applications. Several simple types of adaptation are discussed that have been shown to yield significant usability benefits for desktop systems and mobile applications. Two case studies are presented to illustrate how an AUI can be incorporated into a typical mobile application and the benefits obtained. 
This paper is organized as follows. Section 2 discusses some usability studies highlighting the usability issues associated with mobile applications. Section 3 discusses the benefits of AUIs and how AUI techniques can be used to address these problems. Sections 4 and 5 describe the design and evaluation of two case studies as well as the different forms of adaptation which were incorporated into these systems. Section 6 presents some lessons learned from these case studies and some implications for the design of adaptive systems in the future.

\section{Usability of Mobile Applications}

The importance of mobile phones as a communication and computational device is increasing daily. The number of mobile phone users is increasing exponentially and the usability of mobile applications is becoming a critical factor [3].

Looije et al. [4] maintain that mobile devices currently suffer from a number of usability issues. These usability issues can be grouped into three main categories:

- Technical - which refers to the battery life of the phone, network connectivity and the limited screen size;

- Environmental - which refers to temperature, light conditions, noise, distraction, mobility of the user, cognitive and psychological constraints for the user, competition for attention from other tasks and the need to manipulate objects other than the mobile device; and

- Social - which refers to usability issues relating to privacy, acceptance, adoption, comfort and personalization.

A study on the usability of mobile systems conducted by the Nielsen Norman Group in 2009 in the United Kingdom and the United States showed that only 59\% of all the required tasks were completed successfully. The usability issues identified in this study included [5]:

- $\quad$ Speed - the biggest factor contributing to users performing poorly.

- Screen - the screen size is too small. There is only a limited amount of information that can fit on the screen.

- Typing - typing on mobile devices is hard. An example is logging in because passwords and usernames often contain a combination of digits and letters as well as special characters.

The next section discusses how AUIs can be used to address some of the usability issues identified in this section, especially the limited screen size and interaction mechanisms.

\section{Adaptive User Interfaces}

\subsection{Types of AUIs}

An adaptive user interfaces (AUIs) can be defined as: “... a software artifact that improves its ability to interact with a user by constructing a user model based on 
partial experience with that user."[6] The basic premise behind AUIs is that users are different and therefore have different needs from an interactive system. The system should adapt to the user, rather than forcing the user to adapt to the system. Each user's characteristics and/or past behaviour are modelled in an attempt to adapt to his/her needs and desires.

AUIs can be classified according the input variables which influence adaptation and the types of adaptation effects. The following four variables commonly influence adaptation [4]:

- User: User-based adaptation is commonly employed in AUIs. AUIs can adapt to the user's preferences, knowledge and skills.

- Task: Adaptation according to the user's current task can ensure that the adaptation is relevant and helps users in their current activity.

- System: Adaptation can take place to adjust to differing device capabilities and variables such as network connectivity (in the case of mobile devices).

- Context: Adaptation according to the user's current context can play an important role, particularly in mobile applications.

AUIs can adapt in many different ways in response to the above variables. Several AUIs have been implemented which adapt the UI to suit the individual user [7], provide assistance with routine tasks [8], recommend and filter information based on user preferences and personalize the presentation of information [9].

Three broad classes of adaptation have been identified as suitable for mobile mapbased applications [24]:

- Information: The information selected and the organization of the information can be adapted.

- Visualization: The presentation of information can be adapted.

- User Interface: The user interface can be adapted in a variety of ways, including adaptation of the UI controls and the interaction techniques.

\subsection{Benefits and Shortcomings}

Browne et al. [10] identified a number of high-level potential benefits of AUIs, including improving accuracy and efficiency and aiding in user learning. AUIs have been touted as potential solutions for problems such as information overload and filtering, learning to use complex systems and automated task completion [11]. AUIs also provide the potential to deliver the benefits of customized software at a lower development cost per user [12].

AUIs can provide many potential benefits, but are not without problems. One of the fundamental usability principles identified by Nielsen [13] is user control and freedom. AUIs can be seen as taking control away from the user and putting them at the mercy of the system. Other possible problems and pitfalls include privacy issues, confusion, learnability and obtrusiveness $[7,14,15]$. Some adaptive systems try to make the adaptation process more transparent to users to aid their understanding and to help them develop a more accurate mental model of the system [16].

The following sections describe two case studies investigating the use of AUIs in the domain of mobile map-based applications. Mobile map-based applications were 
chosen as the application domain as these types of systems are widely used and suffer especially from limited screen size and static interfaces. These two case studies were chosen to investigate how an AUI could be incorporated into a mobile map-based application and the benefits obtained. In the first case study, an adaptive system was developed from scratch. In the second case study, an existing system was reengineered to incorporate an AUI based on adaptation requirements identified in a field study.

\section{Case Study: MediaMaps}

MediaMaps was developed as an example of an adaptive mobile map-based visualization (MMV) system. MediaMaps was only developed as an adaptive system and no non-adaptive system was developed, due to the nature of the functionality provided. MediaMaps supports three types of adaptation, namely information, visualization (presentation) and interface adaptation [17]. The design and evaluation of MediaMaps are discussed in the following sections.

\subsection{Functional Requirements}

The functional requirements for MediaMaps were determined by looking at existing MMV systems and interviewing potential users. This process produced the following functional requirements: Capturing and location-tagging of photos, videos and sound recordings; organization of multimedia into collections, based on time and location; lap-based visualization of media collections and individual items; list-based browsing of media collections; searching of collections based on time and location; and saving and loading of views. Figure 1 shows a screenshot of MediaMaps, showing several media collections and items being visualized in a map-based view.

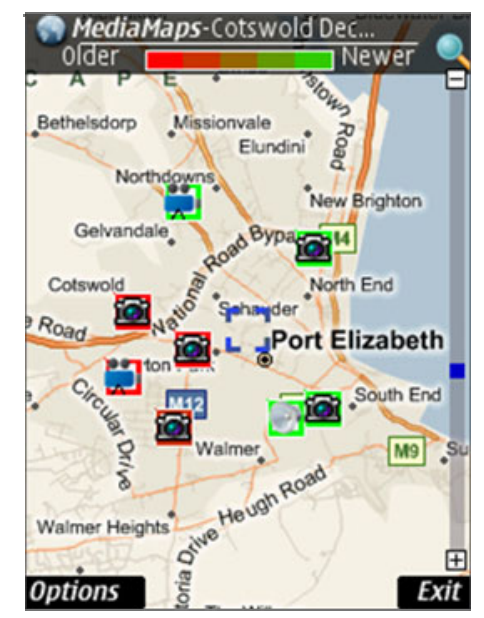

Fig. 1. Screenshot of MediaMaps showing different media collections 


\subsection{Design}

MediaMaps was designed to adapt to the users, their tasks and their context. In order to learn user preferences, a logging mechanism was built into MediaMaps. User interaction is recorded, and various algorithms are used to perform different types of adaptation. The different types of adaptation supported in MediaMaps are briefly discussed below.

Information adaptation is implemented in MediaMaps by sorting media items into media collections based on time and location [18]. The Real-time Event Detection (RED) algorithm was extended to include different media items. The sorting of media items into collections helps to minimize the clutter problem that would otherwise result if a user's entire media collection was visualized in a single view. The grouping of media items is based on time and location. Previous user behavior is used to model the user's media capturing behavior. Media items captured at a similar time and at a similar location are grouped together to form a collection.

Visualization adaptation is implemented in MediaMaps in terms of adapting the visualization to the user's previous behaviour. One the most significant problems of standard zooming and panning techniques is that the user often needs to perform many operations to find information. To address this problem, the visualizations in MediaMaps are adapted in terms of the zoom-level, latitude, longitude, the time period and the current map style (road, satellite photograph or hybrid) [19]. An example of visualization adaptation is shown in Figure 2. The same media collection is shown before adaptation (left) and after adaptation (right). In this example, the zoom-level, map style and location have been adapted based on previous user behaviour.

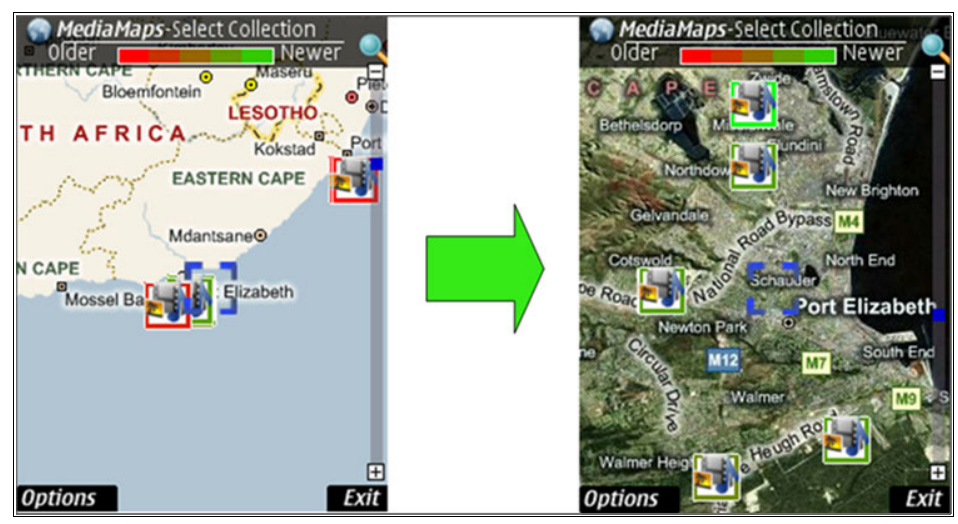

Fig. 2. Visualization adaptation in MediaMaps, before adaptation (left) and after adaptation (right)

Interface adaptation is implemented in the form of list ordering in MediaMaps. These lists include the list of media collections, list of cities and the list of saved views. An example of interface adaptation in MediaMaps is shown in Figure 3. The top section contains the adaptive section of the list, including the most recently used (MRU) option and the two most frequently used (MFU) options [20]. 


\begin{tabular}{|l||}
\multicolumn{1}{|c|}{ Q MediaMaps } \\
Select a Collection \\
\hline At home Aug 2008 \\
Aug 24 \\
Millard Grange Aug 2008 \\
Bethelsdorp Aug 2008 \\
Dogs Aug 2008 \\
Dogs Sep 2008 \\
Humewood Aug 2008 \\
\hline Linton Grange Sep 2008 \\
Fri Sep 26 18:25:48 \\
\hline Masters Sep 2008 \\
Phone Sep 2008 \\
Sound Sep 2008 \\
Summerstrand Aug 2008 \\
\hline Options \\
\hline
\end{tabular}

Fig. 3. Example of interface adaptation in MediaMaps, showing the adaptive section at the top of the list

\subsection{Evaluation}

An international field study of MediaMaps was conducted to evaluate the benefits of the adaptations over a three week period. The following metrics were used to evaluate the three forms of adaptation:

- Accuracy: Information and interface adaptation were evaluated in terms of the accuracy of the adaptations performed. Information adaptation was considered to be accurate when the user accepted a recommendation regarding the sorting of a media item into a media collection. Interface adaptation was regarded as accurate when the user selected an item from the adaptive section of the list.

- User Satisfaction: Several different aspects of user satisfaction were measured, including the general system usability and the usefulness of the three types of adaptation implemented.

User interaction was logged in order to evaluate the accuracy of the information and interface adaptations. A post-test satisfaction questionnaire was used to elicit user satisfaction feedback. International test participants were recruited using a bidding process [21]. Twenty participants (14 male and 6 female) completed the field study. Participants were recruited from twelve countries on four different continents. The participants all had at least three years of mobile phone experience. The results of the evaluation were analyzed in terms of performance and satisfaction metrics and are discussed below.

\subsection{Results}

High levels of accuracy were achieved for the sorting of media items into collections (mean $=87.44 \%)$. A significant correlation co-efficient of 0.79 (p-value $<0.05$ ) was calculated between the accuracy achieved and the number of media items captured. This implied that the accuracy improved as the number of media items captured increased. 
Accuracy information was also calculated for the interface adaptation algorithm. List option selections were split into three categories, namely pre-adaptive selections (when lists were too short to warrant adaptation), adaptive selections and nonadaptive selections. On average, $76.78 \%$ of the selections were selected from the adaptive section of the lists.

Five-point Likert scales were used in the post-test user satisfaction questionnaire, with antonyms at the extremes of the scales. The questionnaire was divided into five sections: Sections B and C related to the general usability of capturing media and searching for media items. Sections D-F evaluated the three forms of adaptation.

User satisfaction results regarding the information, interface and visualization adaptation were very positive. High ratings were obtained for the usefulness of sorting media items into collections (mean=4.20 and median=4.50). The usefulness of the ordering of list options was also highly rated (mean=3.80 and median=4.00). Visualization adaptation was also rated as useful by most of the participants.

The results therefore suggest that the three types of adaptation implemented in MediaMaps were accurate and useful. The next section describes the design of an adaptive mobile tourist guide, called A-POInter, as another example of an adaptive MMV system.

\section{Case Study: A-POInter}

The aim of this case study was to determine the benefits of incorporating an AUI into an existing mobile tourist guide, called POInter [22].

\subsection{Requirements Analysis}

A list of adaptation suggestions for A-POInter was compiled based on typical requirements for a mobile tourist guide [23] and suggested AUI techniques for MMV systems (Section 3.1). A field study was conducted in order to measure the extent to which participants agreed with these suggestions.

The results of the field study clearly showed that the participants would like to use an adaptive mobile tourist guide. Participants generally preferred most frequently used (MFU) selections for most adaptation parameters, including search criteria, filtering search results, reordering menu items, zooming, panning and the selection of map style. This was to be expected since these tasks involve repetitive user interactions with the system.

Most recently used (MRU) was preferred for the selection of a starting map. This was to be expected as this would be useful for tourists needing to quickly access the same area of interest (AOI) that they used the last time. A combination of MFU and MRU was preferred for placing items at the top of the search criteria list.

\subsection{Design}

The design and implementation of the adaptive features in A-POInter were separated into the same three types of adaptation, namely information, visualization and interface adaptation. For the purposes of this paper, only some examples of the adaptive features will be discussed. 
Upon load completion, a user is taken directly to his/her MRU map (Figure 4). If no MRU map exists (e.g. the system is being used for the first time), then the Home Screen will be displayed instead. In POInter tapping "View Map" from the Home Screen would take the user to a screen for selecting a map, or to detect the starting map using GPS. In A-POInter, tapping "View Map" from the Home Screen will immediately visualise the MRU map. If no MRU map exists, tapping "View Map" will take the user to the map selection screen.

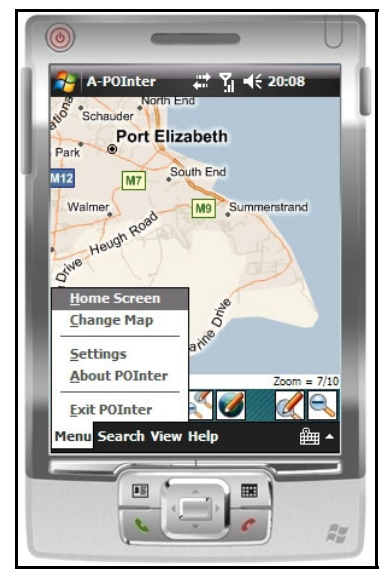

Fig. 4. MRU map with main menu in A-POInter

After a user initiates a search for a category of POIs in A-POInter, the system automatically selects the MFU criteria (Figure 5). Figure 5 also shows the adaptation of list reordering, using the Base Adaptive Partitioning algorithm. The two MFU criteria are selected, but the MRU (3rd item) is not automatically selected.

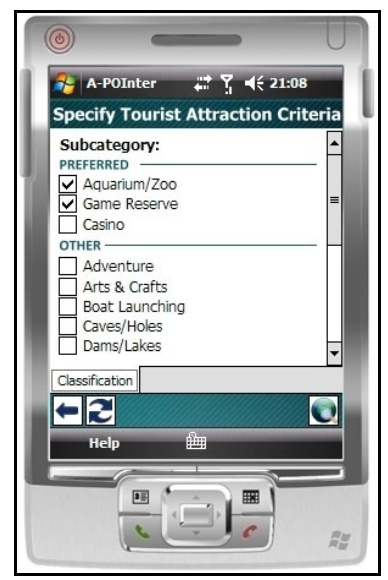

Fig. 5. A-POInter automatically places MFU criteria at the top of the list 
After calculating a set of search results, A-POInter will automatically set the filter percentage to visualise the user's preferred number of search results. After calculating this filter level, the optimal zoom level is calculated and the map is zoomed in or out accordingly (Figure 6).

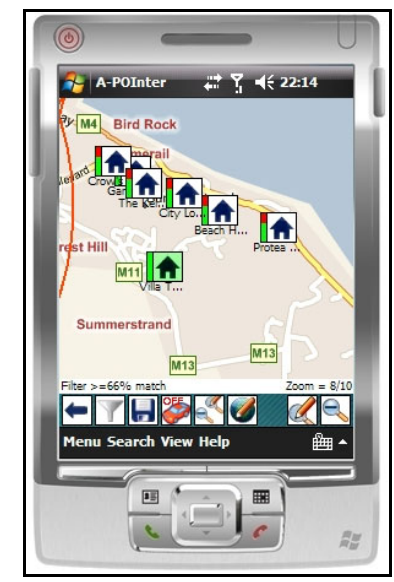

Fig. 6. Search results displayed in A-POInter

\subsection{Evaluation}

An international field study was conducted to determine the usability benefits of A-POInter [25]. The majority of participants were recruited from Mob4Hire [21]. Participants were provided with a test plan, which contained a list of tasks and guidelines regarding the functionality of A-POInter. Tasks included selecting, navigating and manipulating a map, searching for points-of-interest (POIs), viewing and filtering search results, interacting with the search results and using the GPS functionality. The tasks performed were the same tasks used during the requirements field study.

A total of 15 participants successfully completed the study. Twelve participants (80\%) were below the age of $39 ; 13(87 \%)$ were male; six $(40 \%)$ were South African participants and nine $(64 \%)$ were international participants from four different continents. The majority of participants $(93 \%)$ had over five years of general computer experience and 12 participants $(80 \%)$ had experience with a smartphone or PDA (at least one year).

Participants were also asked to complete a post-test satisfaction questionnaire, comprising three sections: Information adaptation, visualization adaptation and interface adaptation. The three sections of the questionnaire were composed of statements scored using a 5-point Likert scale (e.g. 1=Frustrating, 5=Satisfying).

\subsection{Results}

The results for information adaptation were very positive. Participants found it highly noticeable and highly useful that A-POInter automatically selected the MRU map 
(median=5). Participants were generally satisfied with this adaptation feature (median=4). Participants found selecting and browsing maps that had been downloaded to be relatively easy (median=4). Several positive aspects were identified from the users' comments, including ease of use, usefulness and enjoyment (satisfaction).

Similar results were also obtained for visualization and interface adaptation. One participant stated the following regarding reordering of search criteria: "After repeated use, I actually enjoyed A-POInter selecting and moving my preferred search criteria to the top. This prevented me from having to scroll and reselect those criteria each time I searched". High ratings for ease-of-use (median>=4) were obtained for all the key tasks, namely selecting and browsing maps, searching for POIs, and zooming and panning. Participants indicated that they were highly satisfied overall (median=5) with the adaptations provided by A-POInter.

\section{Discussion}

This paper has shown that AUIs can be used to effectively address some of the usability issues affecting mobile applications. The results of the two case studies show that including adaptive features in a mobile map-based application can result in improved usability, usefulness and user satisfaction.

The results of the case studies also show that all three types of adaptation can provide significant usability benefits for mobile applications. Information adaptation can assist in sorting data into collections, displaying relevant data and remembering search preferences. Visualization adaptation can assist in remembering preferred visualization techniques, areas of interest and levels of detail. Interface adaptation can assist in remembering preferred interaction techniques, reordering long lists and menu items.

These adaptation techniques can be included in the design of a new mobile application, such as MediaMaps, or incorporated into an existing mobile application, such as A-POInter. More case studies are needed, however to explore the benefits of AUIs for different types of mobile applications.

\section{Conclusions}

Modern mobile applications suffer from several usability issues, including complex interfaces, poor task support, limited interaction techniques and static navigation. These problems are aggravated on mobile devices due to the limited screen size, speed and network connectivity. This paper has shown that AUIs can be used to address some of these usability issues for mobile map-based applications. The results of the two case studies provide evidence that AUIs can improve the perceived usability, usefulness and satisfaction of mobile applications.

Information, visualization and interface adaptation can also assist in providing personalization and supporting flexibility. User modelling is needed, however, to store user preferences and record user interaction. Existing algorithms can then be used to perform the different types of adaptation. 
Acknowledgements. Acknowledgements are due to Ryan Hill who developed the A-POInter system as part of his Masters research at NMMU in 2009.

\section{References}

1. Al-bar, A., Wakeman, I.: A Survey of Adaptive Applications in Mobile Computing. In: Proc. International Conference on Distributed Computing Systems (ICDCSW 2001), pp. 246-251. IEEE Computer Society, Los Alamitos (2001)

2. Alvarez-Cortes, V., Zayas-Perez, B.E., Zarate-Silva, V.H., Ramirez Uresti, J.A.: Current Trends in Adaptive User Interfaces: Challenges and Applications. In: Proc. the Electronics, Robotics and Automotive Mechanics Conference, pp. 312-317. IEEE Computer Society, Los Alamitos (2007)

3. Hussain, A., Ferneley, E.: Usability metric for mobile application: a goal question metric (GQM) approach. In: Proc. iiWAS 2008, pp. 567-570. ACM, New York (2008)

4. Looije, R., te Brake, G., Neerincx, M.: Usability Engineering for Mobile Maps. In: Proc. International Conference on Mobile Technology, Applications, and Systems (Mobility 2007), pp. 532-539 (2007)

5. Budiu, R.: Overcoming Mobile Web Usability Challenges, http: //www. forum.nokia.com/Technology_Topics /

Design_and_User_Experience/User_Experience/ Overcoming_Mobile_Web_Usability_Challenges.xhtml

6. Langley, P.: User Modeling in Adaptive Interfaces. In: Proc. Seventh International Conference on User Modeling, pp. 357-370. Springer, Heidelberg (1999)

7. Jameson, A.: Adaptive Interfaces and Agents. In: Jacko, A.J., Sears, A. (eds.) HumanComputer Interaction Handbook, pp. 305-330. Erlbaum, Mahwah (2003)

8. Zukerman, I., Albrecht, D.: Predictive Statistical Models for User Modeling. User Modeling and User-Adapted Interaction (UMUAI) 11(1-2), 5-18 (2001)

9. Koelle, D.: Intelligent User Interfaces, http: / / www. davekoelle.com/intint.jsp

10. Browne, D., Norman, M., Riches, D.: Why Build Adaptive Systems? In: Browne, D., Totterdell, P., Norman, M. (eds.) Adaptive User Interfaces, pp. 16-57. Academic Press, London (1990)

11. Höök, K.: Designing and Evaluating Intelligent User Interfaces. In: Proc. the International Conference on Intelligent User Interfaces (IUI 1999), pp. 5-6. ACM Press, New York (1999)

12. Ross, E.: Intelligent User Interfaces: Survey and Research Directions. Department of Computer Science, University of Bristol (2000)

13. Nielsen, J.: Heuristics for User Interface Design, http://www.useit.com/papers/heuristic/heuristic_list.html

14. Opperman, R.: Adaptive User Support: Ergonomic Design of Manually and Automatically Adaptable Software. Lawrence Erlbaum Associates, Hillsdale (1994)

15. Paymans, T.F., Lindenberg, J., Neerincx, M.: Usability Trade-offs for Adaptive User Interfaces: Ease of Use and Learnability. In: Proc. the International Conference on Intelligent User Interfaces (IUI 2004), pp. 301-303. ACM, New York (2004)

16. Kühme, T.: A User-Centered Approach to Adaptive Interfaces. In: Proc. the International Conference on Intelligent User Interfaces (IUI 1993), pp. 243-245. ACM Press, New York (1993) 
17. Van Tonder, B.P., Wesson, J.L.: Design and Evaluation of an Adaptive Mobile Map-Based Visualisation System. In: Proc. IFIP INTERACT 2009, pp. 839-852. Springer, Heidelberg (2009)

18. Chen, W., Chen, M.: Event detection in real time on a personal camera phone image collection. Multimedia Systems (12), 479-492 (2007)

19. Elkan, C.: Naive Bayesian Learning, Department of Computer Science and Engineering. University of California, San Diego (1997)

20. Findlater, L., McGrenere, J.: Impact of Screen Size on Performance, Awareness and User Satisfaction With Adaptive Graphical User Interfaces. In: Proc. SIGCHI Conference on Human Factors in Computing Systems (CHI 2008), pp. 1247-1256. ACM, New York (2008)

21. Mob4Hire. Mob4Hire - Crowdsourced Mobile Application Testing, http: / / www. mob4hire.com

22. Hill, R.T., Wesson, J.L.: Using Mobile Preference-Based Searching to Improve Tourism Decision Support. In: Proc. SAICSIT 2008: Annual Research Conference of the South African Institute of Computer Scientists and Information Technologists, pp. 104-113. ACM, New York (2008)

23. Baus, J., Chreverst, K., Kray, C.: A Survey of Mobile Tourist Guides. In: Map-based Mobile Services: Theories, Methods and Implementations, pp. 197-216 (2005)

24. Cena, F., Console, L., Gena, C., Goy, A., Levi, G., Modeo, S., Torre, I.: Integrating Heterogeneous Adaptation Techniques to Build a Flexible and Usable Mobile Tourist Guide. AI Communications 19(4), 369-384 (2006)

25. Nielsen, C.M., Overgaard, M., Pedersen, M.B., Stage, J., Stenild, S.: It's worth the hassle! the added value of evaluating the usability of mobile systems in the field. In: Proc. NordiCHI 2006, pp. 272-280. ACM, New York (2006) 